\title{
A New Three-Dimensional (3-D) Scalar Finite Element Method to Compute $T_{0}$
}

\author{
Viet Phuong Bui ${ }^{1}$, Yann Le Floch ${ }^{2}$, Gérard Meunier ${ }^{1}$, and Jean-Louis Coulomb ${ }^{1}$ \\ ${ }^{1}$ Laboratoire d'Electrotechnique de Grenoble, UMR 5529 INPG/UJF-CNRS, \\ ENSIEG-BP, 46-38402 Saint Martin D'Hères Cedex, France \\ ${ }^{2}$ CEDRAT, 38246 Meylan, France
}

\begin{abstract}
This paper presents a new computational method of magnetic field in the air created by the known current density distribution in coils or solid conductor. The method consists of decoupling three components of the vector field $T_{0}$. The method is faster and requires less memory than the classical approach, used in the magnetostatic and magnetodynamic finite-element formulations.
\end{abstract}

Index Terms-Finite-element method, magnetic scalar potential, nodal interpolation, source field.

\section{INTRODUCTION}

$\mathbf{T}$ HE FORMULATIONS in magnetic scalar potential $T_{0} \phi$ and $T T_{0} \phi[1]-[3]$ actually provide a general three-dimensional (3-D) finite-element solution for the electrotechnical devices. In these formulations, we need to pre-compute a field $T_{0}$, and many different methods are now available for this manipulation. A new computation of nodal $T_{0}$ with high performance, allowing the implementation of solutions which are compatible with the complex simulations, has been recently developed [2]. It also requires a pre-calculation of the magnetic source field $H$ in the air created by the coils or solid conductor.

However, the calculation of this vector field rapidly becomes significant in time and in memory place for complex devices that are appearing more and more in industry, and strongly limits the interest of the approach of magnetic scalar potential. The aim of this paper is to try reducing these costs.

\section{New Computation of NodAl $T_{0}$}

A nodal $T_{0}$ is defined by giving

$$
T_{0}=H-\operatorname{grad}(\delta \phi)
$$

where

$H$ source field due to coils or solid conductors in the air;

$\delta \phi$ potential increment on the common surface $\Gamma_{01}$ between the regions with reduced scalar potential and total scalar potential, which assures the continuity of the tangential component of magnetic field.

Once the source field $H$ is calculated, the potential increment $\delta \phi$ is easily computed by the finite-element method

$$
(H-\operatorname{grad}(\delta \phi)) \times n=0 \text { on } \Gamma_{01} .
$$

The problem now consists of computing of source field $H$. In fact, once the current density distribution in coils or solid conductor is known, we can simply calculate $H$ by the Biot-Savart formula or by the finite-element method in the entire domain $\Omega$

$$
\operatorname{rot}(H)=j \text { on } \Omega \text {. }
$$

For the finite-element resolution, the vector calculation (1) in the entire domain $\Omega$ must be carried out. It requires an important memory and computation time in the complex problem simulation. It is thus necessary to find a new computation of the source field $H$.

We will show that it is possible to compute economically the source field $H$ by using the scalar calculations instead of the vectorial one. The simplification relies on the decoupling of three components of the vector $H$. Naturally, we realize that the memory and the calculation time can be minimized by considering the following.

- The integration and the assemblage of a scalar quantity instead of a vectorial one. The matrix dimension is thus reduced to 9 .

- The resolution of a three scalar matrices system instead of a vectorial one.

After realizing successively the resolution of each component, the vector source field $H$ will be reconstituted for computing $T_{0}$.

We then developed a computation of source field $H$ by decoupling the calculation of that vector into three calculations of each vectorial component.

\section{New Computation of Source Field $H$}

The problem is simply composed by air and coils in which flow the current density $j_{s}$, enclosed inside the domain $\Omega$ (see Fig. 1).

So we want to resolve

$$
\begin{cases}\operatorname{rot}(H)=j_{s} & \text { on } \Omega \\ \operatorname{div}(H)=0 & \text { on } \Omega \\ H \cdot n=0 & \text { on } \Gamma_{B} \\ H \times n=0 & \text { on } \Gamma_{H}\end{cases}
$$

For finding the weak form of the problem (2), we minimized the functional

$$
F(H)=\int_{\Omega}\left(\left(\operatorname{rot}(H)-j_{s}\right)^{2}+(\operatorname{div}(H))^{2}\right) d \Omega
$$

So

$$
\frac{\partial F(H)}{\partial H}=0 .
$$




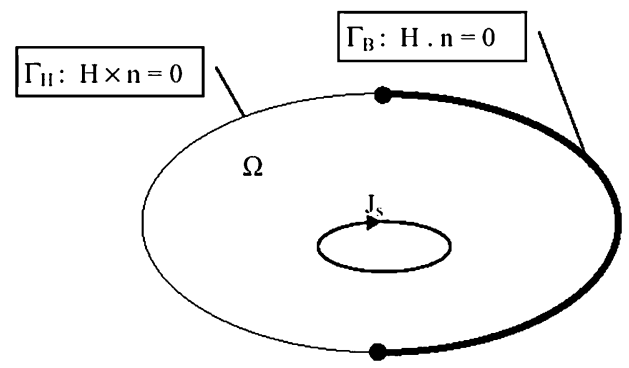

Fig. 1. Problem prototype.

Thus, the weak form is

$$
\begin{aligned}
\int_{\Omega}\left(\frac{\partial \operatorname{rot}(H)^{t}}{\partial H} \operatorname{rot}(H)+\frac{\partial \operatorname{div}(H)}{\partial H}\right. & \operatorname{div}(H)) d \Omega \\
& =\int_{\Omega}\left(\frac{\partial \operatorname{rot}(H)^{t}}{\partial H} j_{s}\right) d \Omega .
\end{aligned}
$$

Then, the finite-element formulations in the Cartesian coordinates could be developed.

We write the discrete form

$$
H=\left(\begin{array}{c}
\sum_{i} w_{i} H_{x i} \\
\sum_{i} w_{i} H_{y i} \\
\sum_{i} w_{i} H_{z i}
\end{array}\right)=\sum_{i} w_{i} H_{i}
$$

in which the couples $\left(w_{i}, H_{i}\right)$ are associated to the nodes $i$ of the finite-element domain.

We thus find to solve (the domain $\Omega$ is entirely homogeneous)

$$
\begin{aligned}
\int_{\Omega}\left(\frac{\partial \operatorname{rot}(H)^{t}}{\partial H_{i}} \operatorname{rot}(H)+\frac{\partial \operatorname{div}(H)}{\partial H_{i}} \operatorname{div}(H)\right) d \Omega \\
=\int_{\Omega}\left(\frac{\partial \operatorname{rot}(H)^{t}}{\partial H_{i}} j_{s}\right) d \Omega .
\end{aligned}
$$

$H_{i}$ represents a set of unknown factors

$$
\left(H_{x 1}, \ldots, H_{x i}, H_{y i}, H_{z i}, \ldots\right) \text {. }
$$

Supposing

$$
\begin{aligned}
& W_{1 i}=\left(\begin{array}{lll}
w_{i} & 0 & 0
\end{array}\right)^{t} \\
& W_{2 i}=\left(\begin{array}{lll}
0 & w_{i} & 0
\end{array}\right)^{t} \\
& W_{3 i}=\left(\begin{array}{lll}
0 & 0 & w_{i}
\end{array}\right)^{t}
\end{aligned}
$$

we obtain, for the matrix term

$$
\begin{aligned}
& \frac{\partial \operatorname{rot}(H)^{t}}{\partial H_{i}} \cdot \operatorname{rot}(H)=\left(\begin{array}{l}
\operatorname{rot}\left(W_{1 i}\right) \cdot \operatorname{rot}(H) \\
\operatorname{rot}\left(W_{2 i}\right) \cdot \operatorname{rot}(H) \\
\operatorname{rot}\left(W_{3 i}\right) \cdot \operatorname{rot}(H)
\end{array}\right) \\
& \frac{\partial \operatorname{div}(H)^{t}}{\partial H_{i}} \operatorname{div}(H)=\left(\begin{array}{l}
\operatorname{div}\left(W_{1 i}\right) \operatorname{div}(H) \\
\operatorname{div}\left(W_{1 i}\right) \operatorname{div}(H) \\
\operatorname{div}\left(W_{1 i}\right) \operatorname{div}(H)
\end{array}\right)
\end{aligned}
$$

and for the source term

$$
\frac{\partial \operatorname{rot}(H)^{t}}{\partial H_{i}} \cdot j_{s}=\left(\begin{array}{c}
\operatorname{rot}\left(W_{1 i}\right) \cdot j_{s} \\
\operatorname{rot}\left(W_{2 i}\right) \cdot j_{s} \\
\operatorname{rot}\left(W_{3 i}\right) \cdot j_{s}
\end{array}\right)
$$

Instead of a vector formulation (3), we then get three scalar, one of which the first component is presented as

$$
\begin{aligned}
\int_{\Omega}\left(\operatorname{rot}\left(W_{1 i}\right) \cdot \operatorname{rot}(H)+\operatorname{div}\left(W_{1 i}\right) \operatorname{div}(H)\right) d \Omega \\
=\int_{\Omega}\left(\operatorname{rot}\left(W_{1 i}\right) \cdot j_{s}\right) d \Omega .
\end{aligned}
$$

We try to decompose the vector $H$ into three components, $H_{x}, H_{y}, H_{z}$, respectively corresponding to each scalar formulation above for our objective.

By using the Green's theorem and the properties of the vector operators, after developing the right term of (4), we obtain an expression of the $H_{x}$ component

$$
\begin{aligned}
\int_{\Omega}\left(\operatorname{rot}\left(W_{1 i}\right) \cdot \operatorname{rot}(H)+\operatorname{div}\left(W_{1 i}\right) \operatorname{div}(H)\right) d \Omega \\
=\int_{\Omega}\left(\operatorname{grad}\left(w_{i}\right) \cdot \operatorname{grad}\left(H_{x}\right)\right) d \Omega \\
\quad-\int_{\Gamma}\left(\left(w_{i} \operatorname{grad}\left(H_{x}\right)\right) \cdot n\right) d \Gamma \\
\quad-\int_{\Gamma}\left(\left(W_{1 i} \times n\right) \cdot \operatorname{rot}(H)+\operatorname{div}(H)\left(n \cdot W_{1 i}\right)\right) d \Gamma .
\end{aligned}
$$

The same process will be executed with the $H_{y}$ and $H_{z}$ components.

We note that there is still the coupling between the components of the vector $H$ in the second surface integral. However, this coupling may vanish according to the type of boundary $\Gamma$.

In case of no symmetric and no periodic problems, we thus consider a boundary $\Gamma$ which encloses the whole domain and throughout which the magnetic field $H$ is zero. In the 3-D finiteelement modeling, we can use a special numerical tool call "infinite box" [4]. So in the scalar formulations preceding, there only remains the volume integral with three components of vector $H$ completely uncoupled

$$
\int_{\Omega}\left(\operatorname{grad}\left(w_{i}\right) \cdot \operatorname{grad}\left(H_{x}\right)\right) d \Omega=\int_{\Omega}\left(\operatorname{rot}\left(W_{1 i}\right) \cdot j_{s}\right) d \Omega .
$$

In the contrary case, thanks to the boundary conditions on $\Gamma$ $\left(H_{t}=0\right.$ or $\left.H_{n}=0\right)$, if the plans of symmetry and periodicity are parallel to the principal plans (XY, YZ, XZ) as shown in Fig. 2, it is easy to show that the surface integrals disappear in the scalar formulations. We then obtain the same formulation as (6). Hence, the computation of the vector source field $H$ with three scalar formulations has been established.

However, if the plans of symmetry or periodicity are not parallel to the principal plans (as shown for most of the cylindrical problems), the coupling always exists. We cannot compute the source field $H$ with three scalar calculations.

Remarkably, in the modeling of 3-D finite element, if the cylindrical problem possesses the plans of symmetry or periodicity, which rotate around an axis, the choice is usually the Z-axis. 


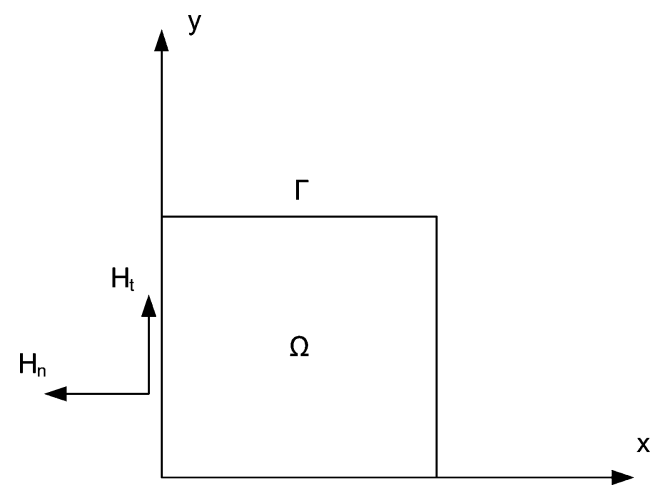

Fig. 2. Parallel boundary.

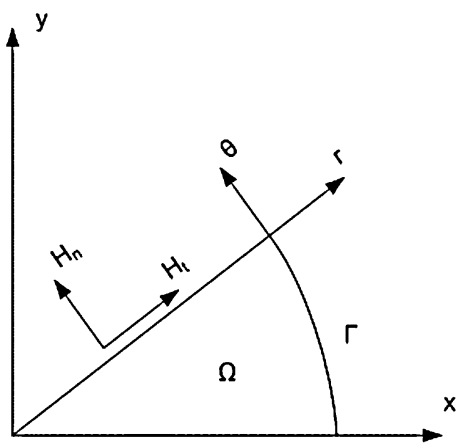

Fig. 3. Cylindrical boundary.

As a result, thanks to the simplification when denoting the components of the source field $H$,follow $H_{t}$ and $H_{n}$ as shown in Fig. 3, we proposed to use the cylindrical coordinates for computing this source field. Unfortunately, we found that the coupling of three components of the vector field $H$ exists even in the volume integral, so we look for another solution.

In fact, a method that gives a partial decoupling was proposed. The computation of the source field $H$ could be carried out with the following.

- The scalar formulation of $H_{z}$ based on the preceding

$$
\int_{\Omega}\left(\operatorname{grad}\left(w_{i}\right) \cdot \operatorname{grad}\left(H_{z}\right)\right) d \Omega=\int_{\Omega}\left(\operatorname{rot}\left(W_{3 i}\right) \cdot j_{s}\right) d \Omega .
$$

- The formulation coupled of $H_{x}$ and $H_{y}$ in which the coupling between two components $H_{x}$ and $H_{y}$ appears only in the surface integral. We thus treat it by using the linear relation of a component on one boundary with components on the other one

$$
H_{1 x y}=[R] H_{2 x y}
$$

in which $R$ is the rotation matrix.

The results obtained from this computation (two formulations) are still very interesting. Indeed, independently of the time calculation required, the memory place becomes rapidly penalized in the industrial vectorial problems.

The proposed formulations (three scalar formulations and formulation coupled) have been implemented in the finite-element code of FLUX3D software and the example presented below proved the efficiency of our proposition.

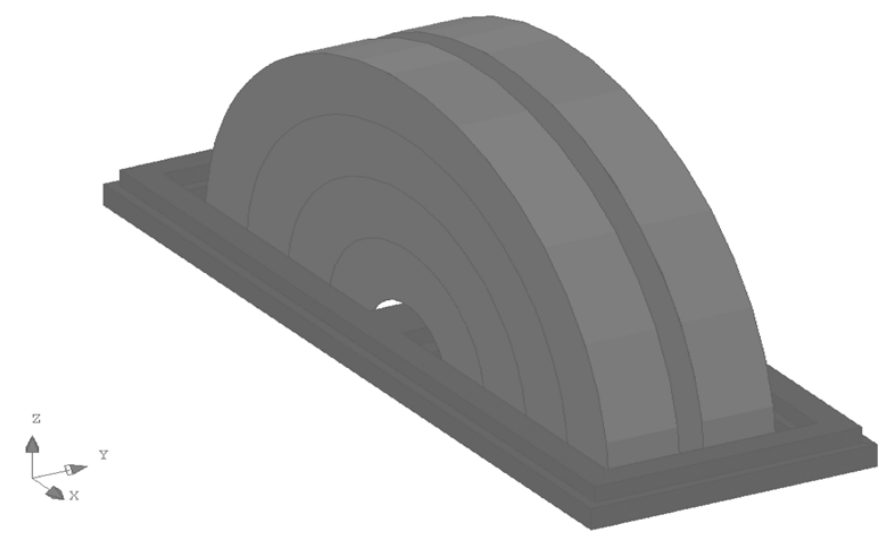

Fig. 4. Geometry of the transformer.

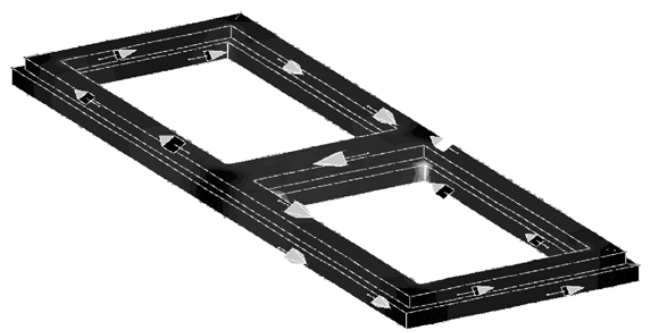

Fig. 5. Induction obtained in the magnetic circuit (three solutions).

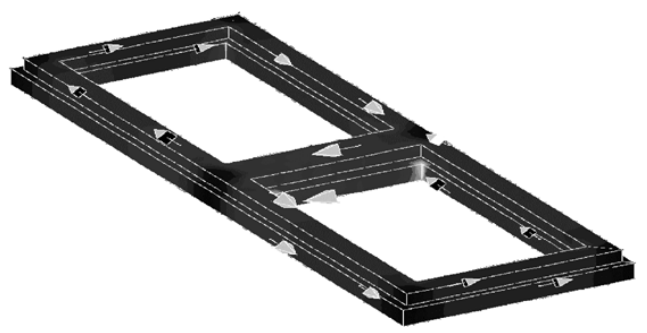

Fig. 6. Induction obtained in the magnetic circuit (standard).

\section{VALIDATION}

The studied example is a current transformer concerning the magnetodynamic problem coupling between electric circuits and multiply connected magnetic circuits. So, we need to use the new $T_{0} \phi$ formulation to solve this problem. In order to satisfy the request of memory capacity in the computation of the nodal $T_{0}$, we inject only one supplied coil instead of eight (the current density is constant along the cross section) as the description of this transformer.

The 3-D finite-element modeling represents a half of the device due to a symmetry of which the plan is parallel to the plan XY (see Fig. 4). The computation of the source field $H$ by three scalar formulations is consequently allowed.

The obtained results have been validated with the help of the resolution using the FLUX3D standard formulation.

After performing the simulations, we have therefore compared our program (three scalar formulations) with the standard formulation for different meshes in regards to the memory place and the calculation time.

This new computation also gives us good results as shown next. 
TABLE I

MEMORY PlaCE IN TWO CASES

\begin{tabular}{c|c|c}
\hline \multirow{2}{*}{$\begin{array}{c}\text { Total amount of } \\
\text { second order nodes }\end{array}$} & \multicolumn{2}{|c}{ Total amount of real (8 bytes) } \\
\cline { 2 - 3 } & Standard & 3 formulations \\
\hline 38553 & 15041889 & 4395007 \\
\hline 81563 & 32049679 & 7992940 \\
\hline 104000 & 40414688 & 10107159 \\
\hline 131795 & Impossible resolution & 12674321 \\
\hline 222374 & Impossible resolution & 21362956 \\
\hline
\end{tabular}

TABLE II

CALCUlation Time in CASES EXeCUted

\begin{tabular}{c|c|c}
\hline \multirow{2}{*}{$\begin{array}{c}\text { Total amount of } \\
\text { second order nodes }\end{array}$} & \multicolumn{2}{|c}{ Calculation time (s) } \\
\cline { 2 - 3 } & Standard & 3 formulations \\
\hline 16279 & 54 & 46 \\
\hline 38553 & 138 & 108 \\
\hline 81563 & 333 & 239 \\
\hline 104000 & 453 & 314 \\
\hline
\end{tabular}

The memory place used with the new formulations is lower than the standard formulation. In fact, our proposition gives the possibility of performing the resolutions with a high range of nodes, which offers a better precision for the results in the given problem, while the standard permits use only with a limited number of nodes (Table I).
The calculation time is also reduced but less significant (Table II). Remarkably, if the eight coils are supplied and we have a great mesh, it could lead to strong improvements in terms of CPU time in comparison with the standard.

\section{CONCLUSION AND PERSPECTIVE}

In this paper, a memory place and calculation time minimization allows us to take into account real devices with more complex geometries. Thanks to the same small matrix systems to solve, an improvement of calculation time which is divided by two or three may occur in comparison with our actual proposition.

\section{REFERENCES}

[1] S. Bouissou, F. Piriou, C. Kieny, and G. Tanneau, "Numerical simulation of a power transformer using 3D finite element method coupled to circuit equation," IEEE Trans. Magn., vol. 30, no. 5, pp. 3224-3227, Sep. 1994.

[2] Y. LeFloch, G. Meunier, C. Guérin, P. Labie, X. Brunotte, and D. Boudaud, "Coupled problem computation of 3-D multiply connected magnetic circuits and electric circuits," IEEE Trans. Magn., vol. 39, no. 3, pp. 1725-1728, May 2003.

[3] G. Meunier, Y. LeFloch, and C. Guérin, "A nonlinear circuit coupled $\mathrm{t}-\mathrm{t}_{0}-\phi$ formulation for solid conductors," IEEE Trans. Magn., vol. 39, no. 3, pp. 1729-1734, May 2003.

[4] X. Brunotte, G. Meunier, and J. F. Imhoff, "Finite element modeling of unbounded problems using transformations: A rigorous, powerful and easy solution," IEEE Trans. Magn., vol. 28, no. 2, pp. 1663-1669, Mar. 1992.

Manuscript received June 20, 2005 (e-mail: Phuong.Bui-Viet@leg.ensieg. inpg.fr). 